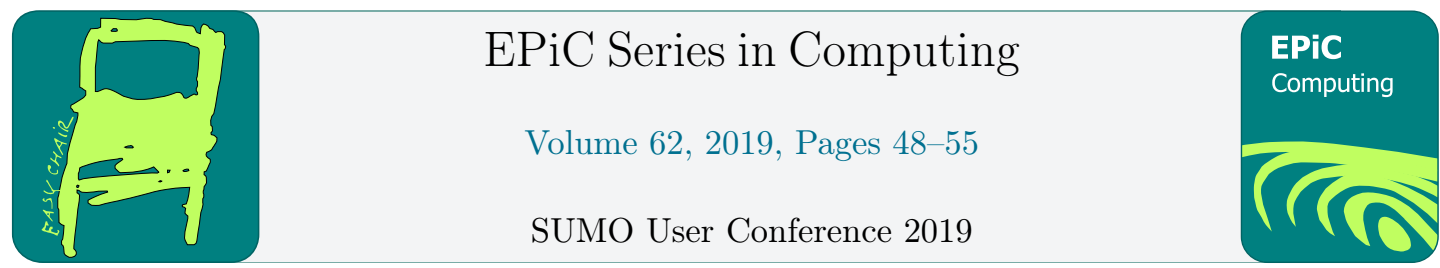

\title{
Testing an Adaptive Cruise Controller with coupled traffic and driving simulations
}

\author{
Mirko Barthauer ${ }^{1}$ and Alexander Hafner ${ }^{2}$ \\ 1 Technische Universität Braunschweig, Institute of Transportation and Urban Engineering, \\ Hermann-Blenk-Straße 42, 38108 Braunschweig, Germany \\ 2 Technische Universität Braunschweig, Institute of Automotive Engineering, \\ Hans-Sommer-Straße 4, 38106 Braunschweig, Germany
}

\begin{abstract}
In many cases, driving simulator studies target how test persons interact with surrounding traffic and with traffic signals. Traffic simulations like SUMO specialize in modeling traffic flow, which includes signal control. Consequently, driving and traffic simulation are coupled to benefit from the advantages of both. This means that all except the driven (ego) vehicle are controlled by the traffic simulation. Essential vehicle dynamics data are exchanged and applied frequently to make the test person interact with SUMO-generated traffic. Additionally, traffic lights are controlled by SUMO and transferred to the driving simulation. The system is used to evaluate an Adaptive Cruise Control (ACC) system, which considers current and future traffic light states. Measures include objective terms like traffic flow as well as the subjective judgement of the signal program, the ACC and the simulation environment.
\end{abstract}

\section{Introduction}

In recent years, researchers from automotive engineering and from traffic engineering have examined some common subjects each from their own perspective. Think of any Advanced Driver Assistance System (ADAS) which impacts following behaviour, lane change or route choice (e.g. [12] [13]). As a consequence, the idea of a common simulation environment has materialised in several organisations. It includes at least a driving simulation and a separate microscopic traffic simulation which are executed in parallel and share their simulation state. At Technische Universität Braunschweig, such a simulation coupling has been developed using SILAB [14] as the driving simulation and SUMO [7] as the traffic simulation part. Here we apply it to evaluate an ACC system for approaching traffic signals. Similar systems have been examined under slightly different conditions: Rittger [10] has evaluated how drivers follow recommendations of a traffic light assistant. Stefanovic et al. [11] have estimated the impact of green light optimised speed advisory (GLOSA) systems on traffic safety and traffic efficiency measures. ACC in general [9]

In first place, some previous works done in the field are reviewed in section 2. Then, the implementation of the simulation coupling is described in detail in section 3. Finally, different

M. Weber, L. Bieker-Walz, R. Hilbrich and M. Behrisch (eds.), SUMO2019 (EPiC Series in Computing, vol. 
aspects of its application are discussed in section 4: Starting from the ACC system and its visualisation to preparing the user experiment and which objectives are expected.

\section{Previous works}

Main findings from literature what to consider for coupling driving and traffic simulation have already been mentioned in [3]. An early draft of the system had already been presented in [3] and [5]. That is why only the most important points are recapitulated here. Viewed from a distance, many simulation couplings share the tasks to reach a realistic driving experience. Unfortunately, some tasks are easier to resolve than others depending on the simulation software. To our knowledge, only the IEEE 1516 standard [1] about the so-called "High-Level Architecture" delivers a blueprint whereafter simulation couplings could be realised. In reality, many constraints lead to custom solutions. There are many formats to describe road networks but both simulations need a common understanding of it to provide coherent vehicle positions. This is especially true when dealing with tortuous road geometry. So either both simulations use a common source or one network gets converted into the other. The other major issue concerns keeping both simulations up-to-date when running them together. Information about vehicles, signal control and other dynamic objects needs to be exchanged frequently and at the right time. The exchange is bidirectional: The ego-vehicle gets transferred to the traffic simulation and surrounding vehicles are placed back in the driving simulation.

\section{Implementation of the simulation coupling}

After giving a general concept of the simulation coupling between SILAB and SUMO in [3], in this paper we provide more information on the proper implementation. The section is structured according to the major points being the road network and the synchronisation part. Finally, some extensions for the ACC system are explained.

For what concerns the road and intersection geometry, driving simulations usually model them more in detail than traffic simulations. In order to avoid any inconsistency when exchanging vehicle positions between the simulations, the road networks have to match closely. Thus the $S I L A B$ road network modules are converted to $S U M O$ network format. Multiple options had been considered to translate the proprietary network description. Finally, we opt for converting to the OpenDrive format first and then use the existing respective import functionality of SUMO. The conversion to OpenDrive format is developed inside the tool chain presented in [6]. In some cases, the resulting $S U M O$ network has to be revised manually to ensure vehicle trajectories match well, especially when carriageways get widened.

As already explained in [3], SILAB can build simulation networks dynamically out of reusable units called modules meanwhile $S U M O$ is bound to a single contigous network. The SILAB approach is a major advantage for professionals designing driving simulation scenarios. So it was no option to forget about it and restrain ourselves to a single module. Instead, all modules are converted and put together in a single $S U M O$ network, placing them next to each other with a predefined offset. Whenever a test person enters a SILAB module, the vehicle positions and angles are transformed between both coordinate systems. This way, SILAB modules can even be rotated to travel through an intersection on a different relation than the first time. SUMO traffic runs within each subnetwork but not across module borders.

In the end, the most challenging part proves to be keeping both simulations synchronised. The Data Processing Unit (DPU) extension of SILAB requests timestep calculations from 
SUMO using TraCI commands, itself being controlled by the central SILAB real-time mechanism. The crucial question is how often should $S U M O$ be called respectively for which timestep duration. Two major options have been considered: Either using a timestep equivalent of the $S I L A B$ frequency or using a longer timestep and call $S U M O$ when the simulation clocks diverge. On one hand, driving simulator frequencies start from $30 \mathrm{~Hz}=\frac{30}{\mathrm{~s}}$ but can reach $100 \mathrm{~Hz}$ or more. On the other hand, SUMO timesteps are limited to a multiple of $1 \mathrm{~ms}$. When using a longer timestep, $S U M O$ vehicle positions need to be extrapolated carefully to move smoothly in the driving simulation. For the time being, the SUMO timestep is aligned with the SILAB frequency and the simulation clocks are checked regularly. This approach does fit our particular case but probably does not scale well with the network size: Given frequencies of at least $30 \mathrm{~Hz}$ to avoid jitter, $S U M O$ has to repeat a lot of calculations which do not necessarily influence the visible area around the ego-vehicle.

During the simulation, several data are exchanged bidirectionally between the simulations as already given in [3]: The ego-vehicle dynamics are copied over to its $S U M O$ equivalent and the surrounding vehicles are inserted or moved inside $S I L A B$. Then, traffic signal states from $S U M O$ are transferred to SILAB. These are considered the main components for urban traffic in the simulation coupling.

There may be further information needed depending on the application. Our ACC system relies on predictions of the next green time for a given signal. Therefore, the signal program structure (sequence of phases) is queried once on startup to initialise a "trivial" signal prediction algorithm for fixed-time signal plans. However, any more sophisticated prediction system ([2] [4]) could be inserted. The traffic light could be made actuated and react to detector events inside $S U M O$.

\section{Case study}

Most driving simulations include a basic traffic simulation themselves, that can move vehicles according to some traffic rules. Usually, fixed-time traffic lights can be operated, too. However, the modeling opportunities are relatively limited in this context compared to specialised simulations like SUMO. With traffic and driving simulations coupled together, infrastructure-related applications can be tested more easily. Different traffic conditions can be recreated well, too.

The study is presented under different aspects. Firstly, the ACC system and the Human Machine Interface (HMI) are presented as a basis. Then, some general differences between traffic simulation and driving simulator studies are discussed and the test network is introduced. Finally, we recall the research questions to be answered in the common driving simulator and traffic simulation study.

\subsection{Adaptive Cruise Control system}

In this work, an ACC system for approaches to signalised intersections is assessed in terms of user acceptance and traffic flow implications. It is based on previous works on ACC for highways (see [9]). Said systems control the longitudinal movement along the current lane. They consider leading vehicles and possibly other constraints like right-of-way rules. The test person needs to steer the ego-vehicle in order to follow its route. This involves lane changes at some intersections to reach the target lane. Additionally, our system gets informed about the current and future traffic signal state it is heading to and shows this information in its HMI display as shown in figure 1. The main decision rules and resulting actions of the ACC system are presented in figure 2: It is only activated when approaching an intersection on a predefined route. The 

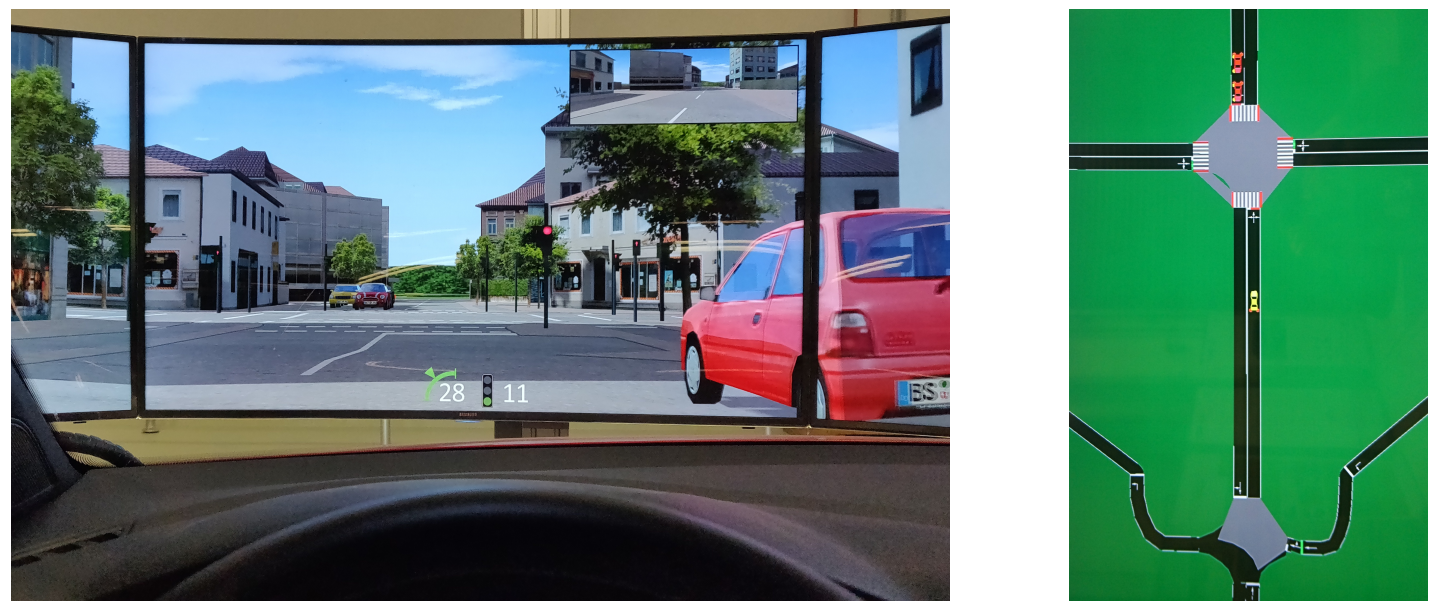

Figure 1: Driver view with the HMI in the simulator (left) and the corresponding SUMO visualisation (right) with the yellow ego-vehicle

vehicle free flow speed in urban environment should be between $30 \mathrm{~km} / \mathrm{h}$ and $50 \mathrm{~km} / \mathrm{h}$. When the vehicle has received the signal state prediction, the expected earliest and latest arrival times at the stop line are estimated. From that, four cases can be differentiated:

1. The signal will remain red throughout the latest arrival time at the stop line. The vehicle will stop.

2. The signal will remain green throughout the latest arrival time at the stop line. The vehicle will pass.

3. The signal will turn green within the arrival time interval. The ACC will lower the speed to make the vehicle arrive at the stop line a few moments after begin of green time.

4. The signal will turn red within the arrival time interval. The ACC will make the vehicle brake gently.

\subsection{Human Machine Interface}

The driving simulator screen shown in figure 1 includes the current HMI of the ACC system under development. It consists of a head-up display with three components from left to right:

- ACC speed in $\mathrm{km} / \mathrm{h}$,

- the future signal state (red or green) the counter refers to and

- the countdown to the future signal state in $s$.

\subsection{General principles of driving simulator studies}

Due to human beings interacting with the driving simulator, researchers tend to use different principles compared to standard traffic simulation studies. In traffic simulation, one simulation run may be interpreted as a sample of a survey. More runs with random traffic situations enlarge 


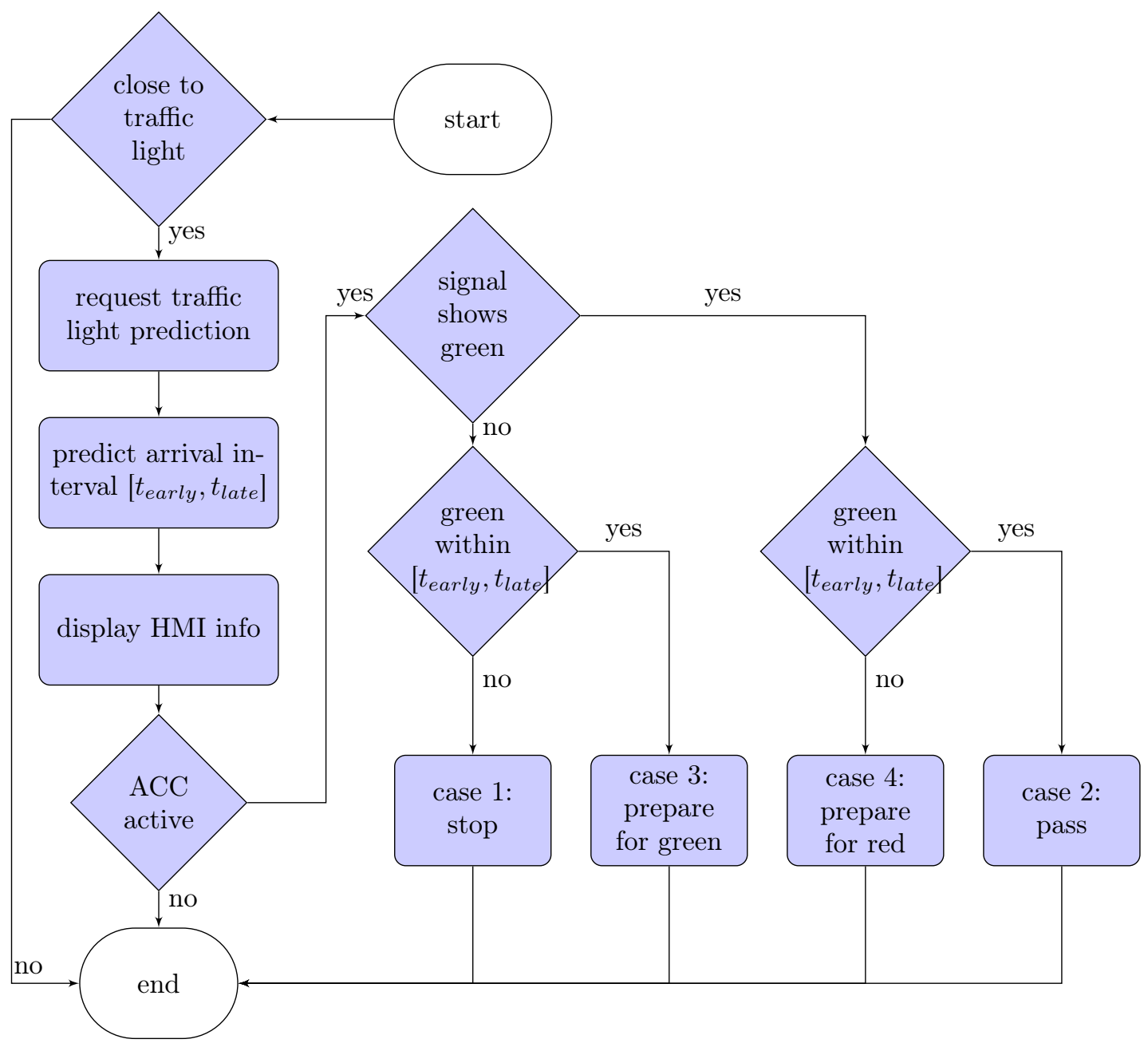

Figure 2: Simplified ACC decision process

the sample which leads to more trustworthy results. In contrast, many driving simulator studies intend to keep many settings constant to recreate the same traffic conditions for every test person. Otherwise, it may be questionable to correlate an effect to an input variable because the conditions when it occurs are not well defined. A very controlled environment can lead to non-realistic surrounding traffic, though [8].

Coming back to the ACC study in question, traffic light states are important. Four different cases for the arrival time at the traffic light are assesssed (see section 4.1). To get meaningful results, we need a guaranteed minimum sample size for every case. For this reason, the SUMO traffic lights are set to a predefined signal phase when the ego-vehicle gets close. The surrounding traffic controlled by $S U M O$ can be queued before entering the road shared with the ego-vehicle, too, to control the test conditions. 


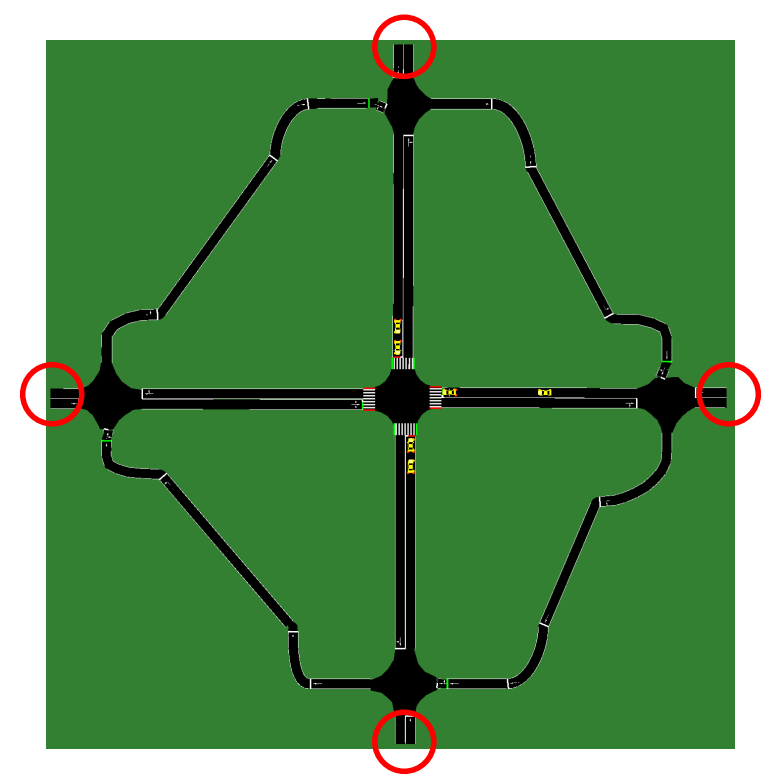

Figure 3: Four-leg example intersection with turning loops in SUMO (red circles indicate inflow/outflow nodes to other modules)

\subsection{Test network}

The driving simulator experiment is still in the planning stage. This means the route is still being composed out of smaller modules according to the following settled principles:

- Turning direction: straight, left and right turning should be included.

- Incoming and outgoing lanes: The intersections vary in size from one to five lanes per approach.

- Signaling: Left turnings may be signalled in a protected or in a permissive manner.

- Traffic: Optionally, traffic may be present in front of the ego-vehicle or in the opposite direction.

One real-world intersection from Braunschweig, Germany, and four synthetic intersections have been modeled to reflect the said principles. A small four-leg intersection is shown in figure 1. Each intersections gets its own SILAB module to recombine them easily. Turning loops like in figure 1 are added to the $S U M O$ version of the network to keep the surrounding traffic going in circles. They can be hidden behind buildings in the driving simulation. At best, the test person should not notice there are vehicles being inserted right before he arrives. Between intersections, SILAB-only sections are placed. They allow the test person to accustom to the simulator, offer some distraction and then to connect differently-sized cross sections.

\subsection{Research questions}

The driving simulator experiment contains two separate phases with respect to the ACC: At first, only the HMI is introduced which makes the driver aware of the next traffic signal timings. 
Later on, the ACC is activated and the driver is merely responsible for controling the lateral movement. Vehicle dynamics and other attributes are recorded during the experiment for further evaluation.

On one hand, the manual driving phase reveals at which speed the driver usually wants to approach urban intersections. On the other hand, it is assessed under which conditions the test persons feel well when supported by the ACC. Approaching a left turn with oncoming traffic is probably a situation where some would prefer to control the vehicle directly.

The current experiment design permits to evaluate traffic flow effects like delays or emissions directly in the traffic simulation for a limited number of situations. With one vehicle equipped with ACC and mostly free-flow conditions, only a subset of interesting scenarios can be tested. However, there is an important outcome for further traffic simulation studies: Distributions of driving behaviour measures like speed under varied conditions can be used to replicate the observed behaviour.

\section{Conclusion and outlook}

Generally speaking, adding a dedicated microscopic traffic simulation to a driving simulator opens the door to new experiments. Traffic simulation models infrastructure and traffic management much more in detail and lets generate random traffic situations easily. This can be used to evaluate ADAS related to signal control like it is being done here: Traffic around the ego-vehicle and the traffic signals are controlled by SUMO meanwhile the driver in SILAB can make use of an ACC which reacts on the traffic signal state. We expect the experiment to indicate under which conditions people accept the ACC-controlled movement and what implications result from it for the surrounding traffic. During the implementation process, it has emerged that mainly synchronising both simulations proves difficult: Either extrapolating $S U M O$ values as increasing the calculation frequency come with downsides. The road network has been converted successfully using the OpenDrive format as intermediate. Overall, the coupling concept still needs some refinement given the issues described above. Nonetheless, the current state already allows for quite some further options to explore: The ACC system could be copied to other vehicles in the traffic simulation, different signal prediction algorithms and actuated signal control could be employed.

\section{Acknowledgments}
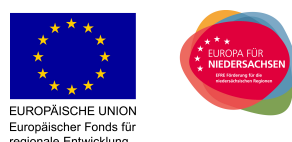

This paper is based on the research project "Simulationskopplung für einen sicheren und nachhaltigen Verkehr" funded by the European Regional Development Fund.

\section{References}

[1] IEEE standard for modeling and simulation (MESS) high level architecture (HLA) : Framework and Rules, volume [Pt. 0] of IEEE standard for modeling and simulation (MESS) high level architecture (HLA) ; [Pt. 0]. Inst. of Electrical and Electronics Engineers, New York, NY, 2000.

[2] Mirko Barthauer and Bernhard Friedrich. Evaluation of a signal state prediction algorithm for car to infrastructure applications. Transportation Research Procedia, 3:982 - 991, 2014. 17th Meeting of the EURO Working Group on Transportation, EWGT2014, 2-4 July 2014, Sevilla, Spain. 
[3] Mirko Barthauer and Alexander Hafner. Coupling traffic and driving simulation: Taking advantage of SUMO and SILAB together. In SUMO 2018-Simulating Autonomous and Intermodal Transport Systems, volume 2 of EPiC Series in Engineering, pages 56-66. EasyChair, 2018.

[4] R. Braun, F. Busch, C. Kemper, R. Hildebrandt, F. Weichenmeier, C. Menig, I. Paulus, and R. Preßlein-Lehle. Lichtsignalsteuerung - TRAVOLUTION - Netzweite Optimierung der Lichtsignalsteuerung und LSA-Fahrzeug-Kommunikation. Straßenverkehrstechnik, 53(6):365-374, 2009.

[5] Alexander Hafner, Mirko Barthauer, and Roman Henze. Kopplung von Fahr- und Verkehrsflusssimulationen für einen sicheren und nachhaltigen Verkehr. In AAET - Automatisiertes und vernetztes Fahren, pages 34-54, 2018.

[6] Alexander Hafner, Adrian Sonka, Roman Henze, and Ferit Küçükay. Integration of the real measurement data into the DVRS. In chassis.tech plus 2016, München, June 2016.

[7] Pablo Alvarez Lopez, Michael Behrisch, Laura Bieker-Walz, Jakob Erdmann, Yun-Pang Flötteröd, Robert Hilbrich, Leonhard Lücken, Johannes Rummel, Peter Wagner, and Evamarie Wießner. Microscopic traffic simulation using SUMO. In The 21st IEEE International Conference on Intelligent Transportation Systems. IEEE, November 2018.

[8] Johan Olstam. Simulation of Surrounding Vehicles in Driving Simulators. PhD thesis, Linköpings Universitet, 2009.

[9] Torben Pawellek, Louisa Liesner, Roman Henze, and Ferit Küçükay. Objektivierungsverfahren für eine kundenoptimale ACC-Systemauslegung. ATZ - Automobiltechnische Zeitschrift, 118(4):74-79, April 2016.

[10] Lena Rittger. Driving Behaviour and Driver Assistance at Traffic Light Intersections. PhD thesis, Universität Würzburg, 2015.

[11] Aleksandar Stevanovic, Jelka Stevanovic, and Cameron Kergaye. Green light optimized speed advisory systems: Impact of signal phasing information accuracy. Transportation research record, (2390):53-67, 2013.

[12] T. Tielert, M. Killat, H. Hartenstein, R. Luz, S. Hausberger, and T. Benz. The impact of trafficlight-to-vehicle communication on fuel consumption and emissions. In 2nd International Internet of Things Conference, IoT 2010; Tokyo; Japan; 29 November 2010 through 1 December 2010. IEEE, Piscataway (NJ), 2010.

[13] SF Varotto, H Farah, B van Arem, and SP Hoogendoorn. Empirical car-following and lanechanging driving behaviour in case of authority transitions between adaptive cruise control and manual driving. In Proceedings of the 2nd TRAIL-beta PhD conference, pages 1-22. Trail-beta, 2016.

[14] WIVW GmbH. Driving Simulation and SILAB. Web page. Accessed 21.02.2018 at https: //wivw.de/en/silab. 